\title{
Electrovacuum geometries in five dimensions
}

\author{
Rafael Ferraro ${ }^{1,2, *}$ \\ ${ }^{1}$ Instituto de Astronomía y Física del Espacio (IAFE, CONICET-UBA), \\ Casilla de Correo 67, Sucursal 28, 1428 Buenos Aires, Argentina. \\ ${ }^{2}$ Departamento de Física, Facultad de Ciencias Exactas y Naturales, \\ Universidad de Buenos Aires, Ciudad Universitaria, Pabellón I, 1428 Buenos Aires, Argentina.
}

\begin{abstract}
The Chong-Cvetič-Lü-Pope $5 D$ rotating charged black hole proves to belong to a set of solutions to Einstein-Maxwell-Chern-Simons (EMCS) equations that share the electromagnetic potential and the Chern-Simons coupling constant but differ in the Kretschmann invariant. This one-parametric family of solutions is found by proposing a properly deformed Plebański-Demiański Ansatz for modeling the metric tensor. While no black-hole solutions for other values of the Chern-Simons coupling constant are found within this Ansatz, another type of non-static electrovacuum solutions to $5 D$ EMCS equations are obtained, namely Kundt spacetimes sourced by a pure-radiation field.
\end{abstract}

\section{INTRODUCTION}

The search for solutions to Einstein equations can be greatly facilitated if the solution is looked for in a family of metrics conveniently prepared. For this aim, one uses an Ansatz whose metrics are simple enough to make easy the control of the Ricci tensor; at the same time they should be sufficiently flexible to have a chance of finding solutions. A well known example of such strategy is the Kerr-Schild (KS) Ansatz [1 3], where the metric is written in the way $g_{\mu \nu}=\stackrel{o}{g}_{\mu \nu}+f(x) k_{\mu} k_{\nu}$; here $\stackrel{o}{g}_{\mu \nu}$ is a "seed" known metric, $k_{\mu}$ is a conveniently chosen null congruence of both metrics $\stackrel{o}{g}_{\mu \nu}$ and $g_{\mu \nu}$, and $f(x)$ is a free function spanning the members of the family. Also Plebański-Demiański (PD) metrics [4] have proved to be a fruitful way of representing the host family of metrics. The components of PD metrics depend on two coordinates $r, p$; two functions $X(p), Y(r)$ play the role of free degrees of freedom; besides they contain several parameters to be identified with the cosmological constant, the angular momenta of the solution, etc. In four dimensions, both KS and PD Ansätze succeed in leading to rotating black-hole solutions. Even the charged Kerr-Newman solution belongs to both Ansätze. Actually PD metrics in four dimensions contain the most general axially symmetric solution to "source-less" Einstein-Maxwell equations. This Petrov type-D solution is characterized by seven parameters: the mass, the Newman-Unti-Tamburino (NUT) charge, the angular momentum, the electric charge, the magnetic charge, the acceleration, and the cosmological constant (see also [5]). For five and higher dimensions there exists a complete catalog of axially symmetric solutions to the vacuum Einstein equations [ 6 [ 8 ]. Instead, the only charged rotating black hole so far obtained is the $5 D$ Chong-Cvetič-Lü-Pope (CCLP) geometry [9], which is a solution to Einstein-Maxwell-Chern-Simons (EMCS) equations for a specific Chern-Simons (CS) coupling constant; it was obtained in the context of five-dimensional minimal gauged supergravity. The CCLP geometry is characterized by the mass, the NUT charge, two angular momenta, the electric charge, and the cosmological constant. There are not clues to build $5 D$ solutions to EMCS equations for other values of the CS coupling constant. However there exist some attempts for the case of equal angular momenta, which resort to numerical [10, 11] or perturbative 12] techniques. Besides, a sector of the $5 D$ Einstein-Maxwell equations has proven to be integrable for a restricted form of the electromagnetic field [13].

We aim to develop a method to search for solutions to $5 D$ EMCS equations, by starting from a proper extension of the 5D PD Ansatz [14]. As a result, we will find that the CCLP solution appears as one among other solutions to EMCS equations for the same electromagnetic potential and CS coupling constant, but differing in the value of the Kretschmann invariant. On the other hand, we will also find a family of $5 D$ geometries conformal to $p p$ waves that are sourced by a pure-radiation electromagnetic field. In Section $\Pi$ we will display the $5 D$ PD Ansatz we will use for introducing the host family of metrics. For these metrics, we will show the eigenvalue-eigenvector structure of the Einstein tensor, and their double Kerr-Schild form. In Section III we will compute the eigenvalue-eigenvector structure of the energy-momentum tensor belonging to a rotating "pointlike" charge. Since the eigenvalue-eigenvector structures of both Einstein and energy-momentum tensors cannot be matched in the PD Ansatz, in Section IV we

\footnotetext{
* Member of Carrera del Investigador Científico (CONICET, Argentina); ferraro@iafe.uba.ar
} 
will introduce an extended PD Ansatz. We will obtain a one-parametric family of solutions accomplishing the EMCS equations for the same electromagnetic potential and Chern-Simons coupling constant $2 \sqrt{G / 3}$, among which the CCLP solution is found. In Section $\nabla$ we will discuss further extensions of the Ansatz; however we will not succeed in getting black-hole solutions for other values of the Chern-Simons coupling constant. In Section VI we will show a different type of non-static solutions to EMCS equations, which is associated with pure-radiation sources. In Section VII we will display the conclusions.

\section{PLEBAŃSKI-DEMIAŃSKI METRICS IN FIVE DIMENSIONS}

We will look for rotating charged black-hole solutions in the set of $5 D$ Plebański-Demiański-like metrics of the form $[14]$

$$
\mathbf{g}=-\frac{Y(r)}{p^{2}+r^{2}} \quad \omega^{0} \otimes \omega^{0}+\frac{X(p)}{p^{2}+r^{2}} \quad \omega^{1} \otimes \omega^{1}+\frac{a^{2} b^{2}}{p^{2} r^{2}} \quad \omega^{2} \otimes \omega^{2}+\left(p^{2}+r^{2}\right) \quad\left(\frac{d r \otimes d r}{Y(r)}+\frac{d p \otimes d p}{X(p)}\right)
$$

where

$$
\begin{aligned}
\omega^{0} & \equiv \frac{\left(1-p^{2} \lambda\right) d t}{\left(1-a^{2} \lambda\right)\left(1-b^{2} \lambda\right)}-\frac{a\left(a^{2}-p^{2}\right) d \phi}{\left(a^{2}-b^{2}\right)\left(1-a^{2} \lambda\right)}-\frac{b\left(b^{2}-p^{2}\right) d \psi}{\left(b^{2}-a^{2}\right)\left(1-b^{2} \lambda\right)}, \\
\omega^{1} & \equiv \frac{\left(1+r^{2} \lambda\right) d t}{\left(1-a^{2} \lambda\right)\left(1-b^{2} \lambda\right)}-\frac{a\left(a^{2}+r^{2}\right) d \phi}{\left(a^{2}-b^{2}\right)\left(1-a^{2} \lambda\right)}-\frac{b\left(b^{2}+r^{2}\right) d \psi}{\left(b^{2}-a^{2}\right)\left(1-b^{2} \lambda\right)} \\
\omega^{2} & \equiv-\frac{\left(1+r^{2} \lambda\right)\left(1-p^{2} \lambda\right) d t}{\left(1-a^{2} \lambda\right)\left(1-b^{2} \lambda\right)}+\frac{\left(a^{2}+r^{2}\right)\left(a^{2}-p^{2}\right) d \phi}{a\left(a^{2}-b^{2}\right)\left(1-a^{2} \lambda\right)}+\frac{\left(b^{2}+r^{2}\right)\left(b^{2}-p^{2}\right) d \psi}{b\left(b^{2}-a^{2}\right)\left(1-b^{2} \lambda\right)}
\end{aligned}
$$

Here, $a, b, \lambda$ are three parameters which can be freely chosen (however, it must be $|a| \neq|b|$ ).

Even though the $5 D$ metrics (1) are deprived of some features of $4 D$ Plebański-Demiański metrics (for instance, they do not contain the parameter associated with the acceleration), the Ansatz (11) should be enough for our purposes. The inverse metric for this Ansatz is

$$
\mathbf{g}^{-1}=-\frac{\mathbf{v}_{0} \otimes \mathbf{v}_{0}}{\left(p^{2}+r^{2}\right) Y(r)}+\frac{\mathbf{v}_{1} \otimes \mathbf{v}_{1}}{\left(p^{2}+r^{2}\right) X(p)}+\frac{\mathbf{v}_{2} \otimes \mathbf{v}_{2}}{p^{2} r^{2}}+\frac{Y(r)}{p^{2}+r^{2}} \frac{\partial}{\partial r} \otimes \frac{\partial}{\partial r}+\frac{X(p)}{p^{2}+r^{2}} \frac{\partial}{\partial p} \otimes \frac{\partial}{\partial p}
$$

where

$$
\begin{aligned}
& \mathbf{v}_{0} \equiv r^{-2}\left(a^{2}+r^{2}\right)\left(b^{2}+r^{2}\right)\left(\frac{\partial}{\partial t}+\frac{a\left(1+r^{2} \lambda\right)}{a^{2}+r^{2}} \frac{\partial}{\partial \phi}+\frac{b\left(1+r^{2} \lambda\right)}{b^{2}+r^{2}} \frac{\partial}{\partial \psi}\right) \\
& \mathbf{v}_{1} \equiv p^{-2}\left(a^{2}-p^{2}\right)\left(b^{2}-p^{2}\right)\left(\frac{\partial}{\partial t}+\frac{a\left(1-p^{2} \lambda\right)}{a^{2}-p^{2}} \frac{\partial}{\partial \phi}+\frac{b\left(1-p^{2} \lambda\right)}{b^{2}-p^{2}} \frac{\partial}{\partial \psi}\right) \\
& \mathbf{v}_{2} \equiv a b\left(\frac{\partial}{\partial t}+\frac{1}{a} \frac{\partial}{\partial \phi}+\frac{1}{b} \frac{\partial}{\partial \psi}\right)
\end{aligned}
$$


The orthogonal bases $\omega^{i}=\left\{\omega^{0}, \omega^{1}, \omega^{2}, d r, d p\right\}$ and $\mathbf{v}_{i}=\left\{\mathbf{v}_{0}, \mathbf{v}_{1}, \mathbf{v}_{2}, \partial / \partial r, \partial / \partial p\right\}$ are dual, except for normalization factors. In fact it is

$$
\omega^{i}\left(\mathbf{v}_{j}\right)=0, \quad \forall i \neq j
$$

They can be normalized:

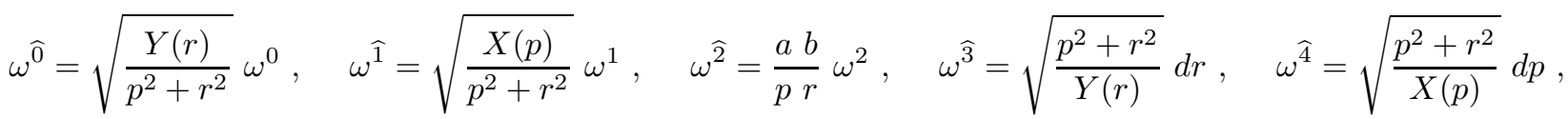

$$
\begin{aligned}
& \mathbf{v}_{\widehat{0}}=\frac{\mathbf{v}_{0}}{\sqrt{\left(p^{2}+r^{2}\right) Y(r)}}, \quad \mathbf{v}_{\widehat{1}}=\frac{\mathbf{v}_{1}}{\sqrt{\left(p^{2}+r^{2}\right) X(p)}}, \quad \mathbf{v}_{\widehat{2}}=\frac{\mathbf{v}_{2}}{p r}, \quad \mathbf{v}_{\widehat{3}}=\sqrt{\frac{Y(r)}{p^{2}+r^{2}}} \frac{\partial}{\partial r}, \quad \mathbf{v}_{\widehat{4}}=\sqrt{\frac{X(p)}{p^{2}+r^{2}}} \frac{\partial}{\partial p} .
\end{aligned}
$$

Some of the characteristics of the $5 D$ Plebański-Demiański metrics (1) are as follows,

i) They are invariant under the change $r^{2} \longleftrightarrow-p^{2}, X \longleftrightarrow Y$.

ii) The metric is Lorentzian only if $X>0$ and $Y>0$, or $X>0$ and $Y<0$ (however $\partial / \partial r$ would be timelike in the second case).

iii) $\left(p^{2}+r^{2}\right) \mathbf{g}^{-1}$ separates into terms depending only on $r$ or $p$. In particular, this implies that Hamilton-Jacobi equation is separable.

iv) In the chart $(t, \phi, \psi, r, p)$ the determinant is

$$
g=\operatorname{det}[\mathbf{g}]=-\frac{p^{2} r^{2}\left(p^{2}+r^{2}\right)^{2}}{\left(a^{2}-b^{2}\right)^{2}\left(1-a^{2} \lambda\right)^{2}\left(1-b^{2} \lambda\right)^{2}} .
$$

v) Einstein tensor $G_{\nu}^{\mu}$ is linear in the functions $X, Y$. In five dimensions only the first and second derivatives of $X$ and $Y$ appear in $G_{\nu}^{\mu}$. Because of this reason the solutions $X, Y$ to vacuum Einstein equations come with additive free constants (see item vii) 1

vi) $\left(p^{2}+r^{2}\right) R$ exhibits separation of variables:

$$
\left(p^{2}+r^{2}\right) R=-p^{-2}\left[p^{2} X^{\prime}(p)-2 a^{2} b^{2} p^{-1}\right]^{\prime}-r^{-2}\left[r^{2} Y^{\prime}(r)+2 a^{2} b^{2} r^{-1}\right]^{\prime} .
$$

vii) The solutions to vacuum Einstein equations are

$$
\begin{gathered}
X_{v a c}(p)=-p^{-2}\left(a^{2}-p^{2}\right)\left(b^{2}-p^{2}\right)\left(1+p^{2} \lambda\right)+\alpha p^{2}+2 n \\
Y_{v a c}(r)=r^{-2}\left(a^{2}+r^{2}\right)\left(b^{2}+r^{2}\right)\left(1-r^{2} \lambda\right)-\alpha r^{2}-2 m
\end{gathered}
$$

where $\alpha, m, n$ are integration constants. This is the Kerr-NUT solution with mass $m$, NUT charge $n$, angular momenta $a, b$, and cosmological constant $\Lambda=6 \lambda$ (however, see item viii) [7, 8, 14, 15]. The integration constant $\alpha$ represents a choice of chart for the Kerr-NUT geometry within the PD Ansatz. The Kretschmann invariant is $\left.R_{\lambda \rho}^{\mu \nu} R_{\mu \nu}^{\lambda \rho}\right|_{v a c}=40 \lambda^{2}+96(m+n)^{2}\left(p^{2}+r^{2}\right)^{-6}\left(3 p^{4}-10 p^{2} r^{2}+3 r^{4}\right)$. Differing from $4 D$, in $5 D$ the mass $m$ and the NUT charge $n$ are not constrained to vanish in the de Sitter geometry: it is enough that $n=-m 2$

\footnotetext{
1 This property could be also obtained in four dimensions by properly redefining $X, Y$.

${ }^{2}$ In five dimensions, $R_{\mu \nu \lambda \rho}-\frac{\Lambda}{6}\left(g_{\mu \lambda} g_{\nu \rho}-g_{\mu \rho} g_{\nu \lambda}\right)$ is affected by a global factor $(m+n)$. Actually, as shown in Ref. [14], for odd dimensions there exists a scaling symmetry making trivial one of the free parameters. Thus, the NUT parameter $n$ could be removed by properly changing coordinates, and the mass $m$ would be completely fixed in the de Sitter geometry.
} 
viii) To guarantee the positiveness of $X_{v a c}, Y_{v a c}$ for the entire range of the coordinates, we can choose $\alpha=0$, and replace $p$ with the coordinate $\theta$ defined as

$$
p^{2}=a^{2} \cos ^{2} \theta+b^{2} \sin ^{2} \theta
$$

i.e.,

$$
\cos ^{2} \theta=\frac{b^{2}-p^{2}}{b^{2}-a^{2}}, \quad \sin ^{2} \theta=\frac{a^{2}-p^{2}}{a^{2}-b^{2}}
$$

By replacing (11) and (12) in the Eq. (9), one sees that $X_{v a c}(\theta)$ will be positive definite if $\lambda, n$ are properly chosen. Besides it is

$$
d p^{2}=\frac{\left(b^{2}-a^{2}\right)^{2} \sin ^{2} \theta \cos ^{2} \theta}{a^{2} \cos ^{2} \theta+b^{2} \sin ^{2} \theta} d \theta^{2} .
$$

ix) $\left\{\mathbf{v}_{i}\right\}=\left\{\mathbf{v}_{0}, \mathbf{v}_{1}, \mathbf{v}_{2}, \partial / \partial r, \partial / \partial p\right\}$ are eigenvectors of the Ricci tensor. This is a very important property of the Plebański-Demiański Ansatz, since it implies that the structure of the Einstein tensor is known independently of the functions $X, Y$. Indeed the functions $X, Y$ only have to do with the eigenvalues of the tensor. Without loss of generality we can write

$$
\begin{gathered}
X(p)=X_{v a c}(p)+f(p), \\
Y(r)=Y_{v a c}(r)+g(r), \\
\left(G_{\nu}^{\mu}+\Lambda \delta_{\nu}^{\mu}\right) v_{i}^{\nu}=\epsilon_{i} v_{i}^{\nu} .
\end{gathered}
$$

Thus $\mathbf{v}_{0}$ and $\partial / \partial r$ share the eigenvalue

$$
\epsilon_{0}=\frac{2 r^{3} f^{\prime}+p\left(p^{2}+3 r^{2}\right) g^{\prime}}{2 p r\left(p^{2}+r^{2}\right)^{2}}+\frac{f^{\prime \prime}}{2\left(p^{2}+r^{2}\right)},
$$

and $\mathbf{v}_{1}$ and $\partial / \partial p$ share the eigenvalue

$$
\epsilon_{1}=\frac{2 p^{3} g^{\prime}+r\left(r^{2}+3 p^{2}\right) f^{\prime}}{2 p r\left(p^{2}+r^{2}\right)^{2}}+\frac{g^{\prime \prime}}{2\left(p^{2}+r^{2}\right)}
$$

therefore it results

$$
\epsilon_{0}+\epsilon_{1}=\frac{p^{-3}\left(p^{3} f^{\prime}\right)^{\prime}+r^{-3}\left(r^{3} g^{\prime}\right)^{\prime}}{2\left(p^{2}+r^{2}\right)}
$$

Besides $\mathbf{v}_{2}$ has the eigenvalue

$$
\epsilon_{2}=\frac{f^{\prime \prime}+g^{\prime \prime}}{2\left(p^{2}+r^{2}\right)}
$$

In terms of the normalized bases (6]), it follows that

$$
\left(G^{\mu}{ }_{\nu}+\Lambda \delta^{\mu}{ }_{\nu}\right) \frac{\partial}{\partial x^{\mu}} \otimes d x^{\nu}=\epsilon_{0} \quad\left(\mathbf{v}_{\widehat{0}} \otimes \omega^{\widehat{0}}+\frac{\partial}{\partial r} \otimes d r\right)+\epsilon_{1}\left(\mathbf{v}_{\widehat{1}} \otimes \omega^{\widehat{1}}+\frac{\partial}{\partial p} \otimes d p\right)+\epsilon_{2} \quad \mathbf{v}_{\widehat{2}} \otimes \omega^{\widehat{2}}
$$

While this result can be considered as the strength of Plebański-Demiański approach, at the same time it is its weakness. In fact, the expression (21) constitutes a severe limitation for the Plebański-Demiański Ansatz as a way to generate solutions to Einstein equations with sources: the equations should be sourced by an energy-momentum tensor with the same eigenvalue-eigenvector structure.

x) Plebański-Demiański metrics can be cast in a Kerr-Schild form that is linear in the function $Y(r)$. Those contributions being inversely proportional to $Y(r)$ are absorbed into a coordinate change. Even more, by allowing for 
complex coordinates, one can also obtain a double Kerr-Schild form that is linear in $X, Y$. In fact, let us perform the complex coordinate change

$$
\begin{aligned}
& d t^{\prime}=d t+\frac{\left(a^{2}+r^{2}\right)\left(b^{2}+r^{2}\right)}{r^{2} Y(r)} d r+i \frac{\left(a^{2}-p^{2}\right)\left(b^{2}-p^{2}\right)}{p^{2} X(p)} d p \\
& d \phi^{\prime}=d \phi-\lambda a d t^{\prime}+\frac{a\left(b^{2}+r^{2}\right)\left(1+r^{2} \lambda\right)}{r^{2} Y(r)} d r+i \frac{a\left(b^{2}-p^{2}\right)\left(1-p^{2} \lambda\right)}{p^{2} X(p)} d p \\
& d \psi^{\prime}=d \psi-\lambda b d t^{\prime}+\frac{b\left(a^{2}+r^{2}\right)\left(1+r^{2} \lambda\right)}{r^{2} Y(r)} d r+i \frac{b\left(a^{2}-p^{2}\right)\left(1-p^{2} \lambda\right)}{p^{2} X(p)} d p \\
& d r^{\prime}=d r, \quad d p^{\prime}=i d p .
\end{aligned}
$$

In the new coordinate basis, the vectors $\mathbf{v}_{i}$ 's look like

$$
\begin{aligned}
& \mathbf{v}_{0}=r^{-2}\left(a^{2}+r^{2}\right)\left(b^{2}+r^{2}\right)\left(\frac{\partial}{\partial t^{\prime}}+\frac{a\left(1-a^{2} \lambda\right)}{a^{2}+r^{2}} \frac{\partial}{\partial \phi^{\prime}}+\frac{b\left(1-b^{2} \lambda\right)}{b^{2}+r^{2}} \frac{\partial}{\partial \psi^{\prime}}\right) \\
& \mathbf{v}_{1}=p^{-2}\left(a^{2}-p^{2}\right)\left(b^{2}-p^{2}\right)\left(\frac{\partial}{\partial t^{\prime}}+\frac{a\left(1-a^{2} \lambda\right)}{a^{2}-p^{2}} \frac{\partial}{\partial \phi^{\prime}}+\frac{b\left(1-b^{2} \lambda\right)}{b^{2}-p^{2}} \frac{\partial}{\partial \psi^{\prime}}\right) \\
& \mathbf{v}_{2}=a b\left(\frac{\partial}{\partial t^{\prime}}+a^{-1}\left(1-a^{2} \lambda\right) \frac{\partial}{\partial \phi^{\prime}}+b^{-1}\left(1-b^{2} \lambda\right) \frac{\partial}{\partial \psi^{\prime}}\right) .
\end{aligned}
$$

Besides it is

$$
\begin{aligned}
\frac{\partial}{\partial r} & =\frac{\partial}{\partial r^{\prime}}+\frac{\mathbf{v}_{0}}{Y(r)} \\
\frac{\partial}{\partial p} & =i \frac{\partial}{\partial p^{\prime}}+i \frac{\mathbf{v}_{1}}{X(p)}
\end{aligned}
$$

Thus, the inverse metric (3) is equal to

$\mathbf{g}^{-1}=\frac{1}{p^{2}+r^{2}}\left[\frac{\partial}{\partial r^{\prime}} \otimes \mathbf{v}_{0}+\mathbf{v}_{0} \otimes \frac{\partial}{\partial r^{\prime}}-\frac{\partial}{\partial p^{\prime}} \otimes \mathbf{v}_{1}-\mathbf{v}_{1} \otimes \frac{\partial}{\partial p^{\prime}}\right]+\frac{\mathbf{v}_{2} \otimes \mathbf{v}_{2}}{p^{2} r^{2}}+\frac{Y(r)}{p^{2}+r^{2}} \frac{\partial}{\partial r^{\prime}} \otimes \frac{\partial}{\partial r^{\prime}}-\frac{X(p)}{p^{2}+r^{2}} \frac{\partial}{\partial p^{\prime}} \otimes \frac{\partial}{\partial p^{\prime}} ;$

so in the complex chart $\left(t^{\prime}, \phi^{\prime}, \psi^{\prime}, r^{\prime}, p^{\prime}\right)$ their components are linear in $X, Y$. The vectors

$$
\begin{aligned}
& \mathbf{k} \equiv-\frac{\partial}{\partial r^{\prime}}=-\frac{\partial}{\partial r}+\frac{\mathbf{v}_{0}}{Y(r)}, \\
& \mathbf{K} \equiv-\frac{\partial}{\partial p^{\prime}}=i \frac{\partial}{\partial p}+\frac{\mathbf{v}_{1}}{X(p)},
\end{aligned}
$$

are null and geodesic whatever the functions $X, Y$ are; besides they are mutually perpendicular. Therefore, the metric (26) has a double Kerr-Schild form, with functions $X, Y$ playing the role of free degrees of freedom in the Kerr-Schild Ansatz (however, in this case they are restricted to depend on a unique coordinate). In particular, the Kerr-NUT metric -whose functions $X, Y$ are given in Eqs. (9) and (10)-can be cast into this form 3

$$
\mathbf{g}_{\text {Kerr-NUT }}^{-1}=\mathbf{g}_{d S}^{-1}-\frac{2 m}{p^{2}+r^{2}} \mathbf{k} \otimes \mathbf{k}-\frac{2 n}{p^{2}+r^{2}} \mathbf{K} \otimes \mathbf{K} .
$$

\section{EINSTEIN-MAXWELL EQUATIONS}

Both the Kerr-Schild and Plebański-Demiański Ansätze have interesting properties regarding Maxwell equations. Let us explain them in the context of the KS Ansatz; the conclusions will be also valid for the double KS form exhibited by PD metrics. Let be the metric [1 3 ]

$$
g_{\mu \nu}=\stackrel{o}{g}_{\mu \nu}+f(x) k_{\mu} k_{\nu}
$$

\footnotetext{
${ }^{3}$ As mentioned in Property (vii), $m=0=n$ is just a particular case of de Sitter geometry. In five dimensions, the geometry is still de Sitter whenever $n$ is equal and opposite to $m$.
} 
where $k_{\mu}$ is a null vector of $\stackrel{o}{g}_{\mu \nu}$. Then, the determinant $g=\operatorname{det}\left(g_{\mu \nu}\right)$ does not depend on $f(x)$, and $k_{\mu}$ is a null vector of $g_{\mu \nu}$ too. Besides, the inverse metric reads

$$
g^{\mu \nu}=\stackrel{o}{g}{ }^{\mu \nu}-f(x) k^{\mu} k^{\nu} .
$$

If $k_{\mu}$ is not only null but geodesic too, i.e.

$$
k^{\mu} k_{\mu}=0 \quad \text { and } \quad 0=k^{\mu}\left(k_{\nu ; \mu}-k_{\mu ; \nu}\right)=k^{\mu}\left(\partial_{\mu} k_{\nu}-\partial_{\nu} k_{\mu}\right),
$$

where $k^{\mu}=\stackrel{o}{g}^{\mu \nu} k_{\nu}=g^{\mu \nu} k_{\nu}$, then it can be proved that if an electromagnetic potential $A_{\mu}=A(x) k_{\mu}$ solves Maxwell equations in the metric $\stackrel{o}{g}_{\mu \nu}$ then it will also solve them in the metric $g_{\mu \nu}$. In fact, it is easy to prove that the field tensor

$$
F^{\mu \nu}=g^{\mu \lambda} g^{\nu \rho}\left(\partial_{\lambda} A_{\rho}-\partial_{\rho} A_{\lambda}\right)
$$

does not depend on the function $f(x)$; so the equations

$$
\partial_{\mu}\left(\sqrt{-g} F^{\mu \nu}\right)=0
$$

are not affected by $f(x)$. Concerning the Einstein-Maxwell problem, this property implies that the Einstein equations will be sourced by an energy-momentum tensor, the electromagnetic energy-momentum tensor

$$
T_{\nu}^{\mu}=-\frac{1}{4 \pi}\left(F^{\mu \rho} F_{\rho \nu}-\frac{1}{4} \delta_{\nu}^{\mu} F^{\lambda \rho} F_{\rho \lambda}\right)
$$

(we use the signature $(-+++\ldots)$ ), which does not contain the unknown function $f(x)$. Moreover, as proved in References [7, 16, 17], if $k^{\mu}$ is tangent to a (null-) geodesic congruence, then $R^{\mu}{ }_{\nu}$ will be linear in $f(x) k^{\mu} k_{\nu}$. So, in terms of the function $f(x)$ the problem gets rather simple, since $f(x)$ appears only linearly in the Einstein tensor. Of course, the success of the Kerr-Schild Ansatz cannot be a priori guaranteed. To have a chance of finding a new solution to Einstein or Einstein-Maxwell equations one should start from a suitable null vector $k^{\mu}$ in order that the sole unknown function $f(x)$ can fulfill the entire set of equations.

In the framework of PD metrics, let us consider the potential of a rotating "pointlike" charge

$$
A_{\mu}=\frac{Q}{p^{2}+r^{2}} k_{\mu}
$$

where $k_{\mu}$ are the covariant components of the null vector (27). We remark that this potential is equivalent to $\mathbf{A}=Q\left(p^{2}+r^{2}\right)^{-1} \omega^{0}$ and $\mathbf{A}=Q\left(p^{2}+r^{2}\right)^{-1} \omega^{1}$, since they differ in pure gauge terms 4 The field $\mathbf{F}=d \mathbf{A}$ verifies the Maxwell equations in the metric (10) whatever the functions $X(p), Y(r)$ are. By computing the eigenvalues and eigenvectors of the energy-momentum tensor (35) it follows that

$$
8 \pi G T_{\nu}^{\mu} \frac{\partial}{\partial x^{\mu}} \otimes d x^{\nu}=-\frac{4 G Q^{2}}{\left(p^{2}+r^{2}\right)^{3}}\left[\mathbf{v}_{\widehat{0}} \otimes \omega^{\widehat{0}}+\frac{\partial}{\partial r} \otimes d r-\mathbf{v}_{\widehat{1}} \otimes \omega^{\widehat{1}}-\frac{\partial}{\partial p} \otimes d p+\frac{p^{2}-r^{2}}{p^{2}+r^{2}} \mathbf{v}_{\widehat{2}} \otimes \omega^{\widehat{2}}\right]
$$

We will try to combine this result with the one in Eq. (21), to know whether there is a chance of getting a solution to $5 D$ Einstein-Maxwell equations within the Ansatz (11), (14), and (15). To this aim, we should find functions $f(p)$, $g(r)$ such that the eigenvalues (17],20) become equal to the ones in the energy-momentum tensor of Eq. (37). By comparing Eqs. (21) and (37) we should search for the eigenvalues

$$
\epsilon_{0}=-\epsilon_{1}=-\frac{4 G Q^{2}}{\left(p^{2}+r^{2}\right)^{3}}, \quad \epsilon_{2}=-\frac{4 G Q^{2}\left(p^{2}-r^{2}\right)}{\left(p^{2}+r^{2}\right)^{4}}
$$

The condition $\epsilon_{0}+\epsilon_{1}=0$, which results from the expected eigenvalues (38), implies $f^{\prime}=-B p+C p^{-3}$ and $g^{\prime}=B r+D r^{-3}$ in Eq. (19). However, these functions $f(p), g(r)$ do not lead to the expected eigenvalues. Therefore, there is not a solution sourced by the rotating "pointlike" charge potential (36) within the Ansatz (11) 5

\footnotetext{
${ }^{4}$ Notice that $k_{\mu} d x^{\mu}=-\left(p^{2}+r^{2}\right) Y(r)^{-1} d r+\omega^{0}$. Besides, from definitions (2), it is easy to verify that $\left(p^{2}+r^{2}\right)^{-1}\left(\omega^{0}-\omega^{1}\right)$ is a closed 1-form.

${ }^{5}$ The static case is not included here because the Ansatz prevents the simultaneous vanishing of $a$ and $b$ (see the determinant 17).
} 


\section{EXTENDING THE PLEBAŃSKI-DEMIAŃSKI ANSATZ}

As a way to enlarge the set of Plebański-Demiański metrics, and thus improve the chance of getting solutions to Einstein-Maxwell equations, we could try including a "conformal" factor in the four-dimensional sector where the electromagnetic field manifests itself (notice that $\left.F_{\mu \nu} v_{2}^{\nu}=0\right) 6$

$$
\mathbf{g}=\Theta(r, p)\left[-\frac{Y(r)}{p^{2}+r^{2}} \quad \omega^{0} \otimes \omega^{0}+\frac{X(p)}{p^{2}+r^{2}} \quad \omega^{1} \otimes \omega^{1}+\left(p^{2}+r^{2}\right) \quad\left(\frac{d r \otimes d r}{Y(r)}+\frac{d p \otimes d p}{X(p)}\right)\right]+\frac{a^{2} b^{2}}{p^{2} r^{2}} \quad \omega^{2} \otimes \omega^{2} .
$$

The potential (36) will still satisfy Maxwell equations, and its energy-momentum tensor will scale with $\Theta(r, p)^{-2}$ :

$$
T_{\nu}^{\mu} \longrightarrow \Theta(r, p)^{-2} T_{\nu}^{\mu}
$$

which means that the so-modified energy-momentum tensor still possesses the structure displayed in Eqs. (37). On the other hand, to save the structure of $G^{\mu}{ }_{\nu}$ displayed in Eq. (21), we are compelled to employ the function $\Theta(r, p)=$ $\left(B p^{2} r^{2}+C\right)^{-2}(B, C$ are integration constants). However, it is not possible to find a set $B, C, X(p), Y(r)$ matching the eigenvalues of the source (37)-(40) 7

So, we should consider other ways of relaxing the Ansatz (1) in order that a source like (37) makes sense in Einstein equations. Let us remark that the source (37) requires the vanishing of $R_{n}^{r}$. If the metric depends on only two coordinates $r, p$, and it is diagonal in the block $(r, p)$, then $R_{r p}$ has the form [20]

$$
\begin{aligned}
R_{r p}= & \frac{1}{2}\left[\log \left(-g^{-1} g_{r r} g_{p p}\right)\right]_{, r p} \\
& -\frac{1}{4}\left[\log g_{r r}\right]_{, p} \quad\left[\log \left(-g^{-1} g_{p p}\right)\right]_{, r}-\frac{1}{4}\left[\log g_{p p}\right]_{, r} \quad\left[\log \left(-g^{-1} g_{r r}\right)\right]_{, p}+\frac{1}{4} g_{, r}^{\mu \nu} g_{\mu \nu, p} .
\end{aligned}
$$

Besides, if $g_{r r}=\left(p^{2}+r^{2}\right) Y(r)^{-1}$ and $g_{p p}=\left(p^{2}+r^{2}\right) X(p)^{-1}$ one obtains

$$
R_{r p}=-\frac{1}{2}\left[\log \left(-g\left(p^{2}+r^{2}\right)^{3}\right)\right]_{, r p}-\frac{1}{2\left(p^{2}+r^{2}\right)}\left[p[\log (-g)]_{, r}+r[\log (-g)]_{, p}\right]+\frac{1}{4} g_{, r}^{\mu \nu} g_{\mu \nu, p} .
$$

So, the simplest way of changing the Ansatz for the metric, while keeping the value $R_{r p}=0$, is

i) keep the form of the block $(r, p)$,

ii) modify the block $(t, \phi, \psi)$ without affecting the values of $g$ and $g^{\mu \nu}{ }_{, r} g_{\mu \nu, p}$.

The preservation of $R_{r p}$ is just one of the clues it should be observed to have a chance of success in getting a solution to Einstein-Maxwell equations. Of course, we also should care that this way of relaxing the Ansatz has not a destructive impact on $F^{\mu \nu}$; otherwise we would affect the fulfilling of Maxwell equations, or the form of $T_{\nu}^{\mu}$. Taking these considerations into account, we will extend the Plebański-Demiański Ansatz by replacing $\omega^{2}$ in the metric (1) with

$$
\mathbf{\Omega}^{2}=\omega^{2}-\frac{p^{2} r^{2}}{a b\left(p^{2}+r^{2}\right)}\left(\mathcal{Y}(r, p) \omega^{0}+\mathcal{X}(r, p) \omega^{1}\right)
$$

where $\mathcal{X}(r, p), \mathcal{Y}(r, p)$ are functions to be chosen. The so-extended Plebański-Demiański Ansatz is then

$$
\mathbf{g}=-\frac{Y(r)}{p^{2}+r^{2}} \omega^{0} \otimes \omega^{0}+\frac{X(p)}{p^{2}+r^{2}} \omega^{1} \otimes \omega^{1}+\frac{a^{2} b^{2}}{p^{2} r^{2}} \mathbf{\Omega}^{2} \otimes \mathbf{\Omega}^{2}+\left(p^{2}+r^{2}\right)\left(\frac{d r \otimes d r}{Y(r)}+\frac{d p \otimes d p}{X(p)}\right) .
$$

Notice that the determinant (7) is effectively preserved, in spite of the modifications introduced in the block $(t, \phi, \psi)$. In fact, the volume associated with $\mathbf{g}$ is

$$
\text { volume }=\frac{a b}{p r} \omega^{0} \wedge \omega^{1} \wedge \Omega^{2} \wedge d r \wedge d p=\frac{a b}{p r} \omega^{0} \wedge \omega^{1} \wedge \omega^{2} \wedge d r \wedge d p
$$

\footnotetext{
6 This kind of metric has been used in Ref. [18] to obtain magnetic dipole-charged solutions with electric charge in five-dimensional minimal supergravity.

7 Within this context, we remark the existence of a non-flat vacuum solution: if $\lambda=0$, then the Ricci tensor is zero for $C=0$, $X_{v a c}(p)=\alpha p^{2}+\beta p^{4}-a^{2} b^{2} B^{2} p^{6}$, and $Y_{v a c}(r)=-\alpha r^{2}+\gamma r^{4}+a^{2} b^{2} B^{2} r^{6}$ ( $\alpha, \beta, \gamma$ are integration constants). This solution is intrinsically curved, since the Riemann tensor cannot be made zero by choosing the integration constants (for other Ricci-flat solutions of this sort, see Ref. [19]). Instead, its $4 D$ analog -which is $\lambda=0, \Theta=(B p r)^{-2}, X_{\text {vac }}(p)=\alpha p^{2}+\beta p^{4}, Y_{v a c}(r)=-\alpha r^{2}+\beta r^{4}-$ is the flat spacetime for any values of the integration constants $\alpha, \beta$.
} 
then the volume does not depend on the "deformations" $\mathcal{X}(r, p), \mathcal{Y}(r, p)$. Besides we will require that $g^{\mu \nu}{ }_{, r} g_{\mu \nu, p}$ keeps its "undeformed" value, which is

$$
g_{, r}^{\mu \nu} g_{\mu \nu, p}=-\frac{4}{p r}-\frac{8 p r}{\left(p^{2}+r^{2}\right)^{2}}
$$

this requirement implies that $\mathcal{X}, \mathcal{Y}$ must be

$$
\mathcal{X}=h(p) \quad \text { and } \quad \mathcal{Y}=q(r)
$$

or

$$
\mathcal{X}=\frac{p^{2}+r^{2}}{p^{2} r^{2}}\left(p^{2} h(r)-a b\right) \quad \text { and } \quad \mathcal{Y}=\frac{p^{2}+r^{2}}{p^{2} r^{2}}\left(r^{2} q(p)-a b\right)
$$

We will choose the first option because it guarantees the separability of $\left(p^{2}+r^{2}\right) \mathbf{g}^{-1}$.

It is worth noticing that the CCLP metric [9, 15] belongs to this Ansatz; it is the case

$$
X_{C C L P}(p)=X_{v a c}(p), \quad Y_{C C L P}(r)=Y_{v a c}(r)+\frac{Q^{2}+2 a b Q}{r^{2}}, \quad \mathcal{X}_{C C L P}=0, \quad \mathcal{Y}_{C C L P}=\frac{Q}{r^{2}}
$$

It could be said that the CCLP metric is somehow "biased" towards the $Y, \mathcal{Y}$ sector. Hopefully, we might find a solution to Einstein-Maxwell equations by "unbiasing" the choice of $\mathcal{X}, \mathcal{Y}$.

As can be seen in Eq. (43), the basis of $\omega^{i}$ 's is no longer orthogonal. Instead the basis $\left\{\omega^{0}, \omega^{1}, \Omega^{2}, d r, d p\right\}$ is orthogonal in the metric (43). Not surprisingly, the inverse metric also has the Plebański-Demiański structure, but vectors $\mathbf{v}_{0}, \mathbf{v}_{1}$ must be, respectively, substituted for

$$
\begin{aligned}
& \mathbf{V}_{0}=\mathbf{v}_{0}+\mathcal{Y}(r, p) \mathbf{v}_{2}, \\
& \mathbf{V}_{1}=\mathbf{v}_{1}+\mathcal{X}(r, p) \mathbf{v}_{2} .
\end{aligned}
$$

The inverse metric reads

$$
\mathbf{g}^{-1}=-\frac{\mathbf{V}_{0} \otimes \mathbf{V}_{0}}{\left(p^{2}+r^{2}\right) Y(r)}+\frac{\mathbf{V}_{1} \otimes \mathbf{V}_{1}}{\left(p^{2}+r^{2}\right) X(p)}+\frac{\mathbf{v}_{2} \otimes \mathbf{v}_{2}}{p^{2} r^{2}}+\frac{Y(r)}{p^{2}+r^{2}} \frac{\partial}{\partial r} \otimes \frac{\partial}{\partial r}+\frac{X(p)}{p^{2}+r^{2}} \frac{\partial}{\partial p} \otimes \frac{\partial}{\partial p} .
$$

Thus $\left\{\mathbf{V}_{0}, \mathbf{V}_{1}, \mathbf{v}_{2}, \partial / \partial r, \partial / \partial p\right\}$ is an orthogonal basis in the tangent space that is dual to $\left\{\omega^{0}, \omega^{1}, \boldsymbol{\Omega}^{2}, d r, d p\right\}$, except for normalization factors.

We are left with the unknowns $h(p), q(r), X(p)$, and $Y(r)$. If the extended Plebański-Demiański Ansatz is useful, we will success in finding the functions $h, q, X, Y$ endowing the Einstein tensor with a suitable eigenvalue-eigenvector structure to be sourced by the energy-momentum tensor belonging to a rotating "pointlike" charge. So let us now turn to the electromagnetic potential (36), where $k_{\mu}$ is still the one described in Footnote 4 , its contravariant version is the vector $\mathbf{k}$ in Eq. (27) where $\mathbf{v}_{0}$ must be replaced with $\mathbf{V}_{0}$. $\mathbf{k}$ is a null and geodesic vector in the extended metric (43) too. As was already pointed out, the potential (36) is gauge equivalent to $\mathbf{A}=Q\left(p^{2}+r^{2}\right)^{-1} \omega^{0}$ and $\mathbf{A}=Q\left(p^{2}+r^{2}\right)^{-1} \omega^{1}$. This equivalence reflects in the "unbiased" 2 -form field

$$
\mathbf{F}=d \mathbf{A}=\frac{2 Q}{\left(p^{2}+r^{2}\right)^{2}}\left(r \omega^{0} \wedge d r+p \omega^{1} \wedge d p\right)
$$

The contravariant field $F^{\mu \nu}$ in the metric (43) is

$$
F^{\mu \nu} \frac{\partial}{\partial x^{\mu}} \wedge \frac{\partial}{\partial x^{\nu}}=\frac{2 Q}{\left(p^{2}+r^{2}\right)^{3}}\left[r \mathbf{V}^{0} \wedge \frac{\partial}{\partial r}-p \quad \mathbf{V}^{1} \wedge \frac{\partial}{\partial p}\right]
$$

The presence of vectors $\mathbf{V}_{0}, \mathbf{V}_{1}$ implies that the extended Ansatz (43) affects $F^{\mu \nu}$ by introducing new terms. Even so, the energy-momentum tensor (35) associated to $F^{\mu \nu}$,

$$
8 \pi G T_{\nu}^{\mu} \frac{\partial}{\partial x^{\mu}} \otimes d x^{\nu}=-\frac{4 G Q^{2}}{\left(p^{2}+r^{2}\right)^{3}}\left[\mathbf{V}_{\widehat{0}} \otimes \omega^{\widehat{0}}+\frac{\partial}{\partial r} \otimes d r-\mathbf{V}_{\widehat{1}} \otimes \omega^{\widehat{1}}-\frac{\partial}{\partial p} \otimes d p+\frac{p^{2}-r^{2}}{p^{2}+r^{2}} \mathbf{V}_{\widehat{2}} \otimes \mathbf{\Omega}^{\widehat{2}}\right]
$$


(we are involving the normalized versions (6) of the orthogonal bases in the tangent and cotangent spaces) exhibits an eigenvalue structure which does not depend on the choice of $\mathcal{X}, \mathcal{Y}$. Instead, the choice of $\mathcal{X}, \mathcal{Y}$ does affect the fulfillment of Maxwell equations. However, by choosing the functions $h(p), q(r)$ in Eq. (46) as

$$
\mathcal{X}(r, p)=\frac{\mu_{\mathcal{X}} Q}{p^{2}}+\zeta, \quad \mathcal{Y}(r, p)=\frac{\mu_{\mathcal{Y}} Q}{r^{2}}-\zeta,
$$

we obtain that $F^{\mu \nu}$ fulfills Maxwell-Chern-Simons equations,

$$
\partial_{\mu}\left(\sqrt{-g} F^{\mu \nu}\right)=\frac{1}{4}\left(\mu_{\mathcal{X}}-\mu \mathcal{Y}\right) \epsilon^{\nu \lambda \rho \alpha \beta} F_{\lambda \rho} F_{\alpha \beta},
$$

with an arbitrary coupling constant proportional to $\mu_{\mathcal{X}}-\mu_{\mathcal{Y}}$ (the value of $\zeta$ is irrelevant, not only at this stage but for the rest of the analysis as well.) 8

The potential (36) satisfies Eq. (56) whatever the functions $X(p)$ and $Y(r)$ are. So we are now left with two unknowns, $X(p)$ and $Y(r)$. Our aim is to properly choose them to obtain solutions to Einstein-Chern-Simons equations with arbitrary Chern-Simons coupling constant.

Concerning the structure of $G^{\mu}{ }_{\nu}+\Lambda \delta^{\mu}{ }_{\nu}$ in the extended Plebański-Demiański Ansatz, one can verify that $\partial / \partial r$ and $\partial / \partial p$ are still eigenvectors whatever $X, Y$ are. As a necessary condition to match the energy-momentum tensor (54), their respective eigenvalues $\epsilon_{3}, \epsilon_{4}$ should be equal and opposite. Without loss of generality, let us we write

$$
\begin{gathered}
X(p)=X_{v a c}(p)+f(p), \\
Y(r)=Y_{v a c}(r)+g(r) ;
\end{gathered}
$$

then one gets that the eigenvalues are effectively equal and opposite if and only if

$$
p^{-3}\left(p^{3} f^{\prime}\right)^{\prime}+r^{-3}\left(r^{3} g^{\prime}\right)^{\prime}=0 .
$$

This linear equation has independent inverse square homogeneous solutions for $f$ and $g 9$ which implies two new constants in $X, Y$. For convenience, we will write the solutions in the following way:

$$
\begin{gathered}
X(p)=X_{v a c}(p)-\mu_{\mathcal{X}} Q \frac{\mu_{\mathcal{X}} Q+2 a b+\beta_{X}}{p^{2}}, \\
Y(r)=Y_{v a c}(r)+\mu_{\mathcal{Y}} Q \frac{\mu_{\mathcal{Y}} Q+2 a b+\beta_{Y}}{r^{2}} .
\end{gathered}
$$

So far, the results for $X, Y$ do not differ from the ones we would have obtained in the previous Section. However we have changed the geometry, by extending the Plebański-Demiański Ansatz, as an attempt to match the eigenvalues and eigenvectors of $G^{\mu}{ }_{\nu}+\Lambda \delta^{\mu}{ }_{\nu}$ with those of the source (54). In fact, the extended Ansatz replaced vectors $\mathbf{v}_{0}, \mathbf{v}_{1}$ with $\mathbf{V}_{0}, \mathbf{V}_{1}$ as defined in Eqs. (49), (50), (55). Thus we obtain that not only $\partial / \partial r, \partial / \partial p$ but $\mathbf{V}_{0}, \mathbf{V}_{1}, \mathbf{v}_{2}$ are eigenvectors of $G^{\mu}{ }_{\nu}+\Lambda \delta_{\nu}^{\mu}$ with eigenvalues

$$
\begin{aligned}
-\epsilon_{0} & =-\epsilon_{3}=\epsilon_{1}=\epsilon_{4}=\frac{3 Q^{2}\left(\mu_{\mathcal{X}}-\mu_{\mathcal{Y}}\right)^{2}}{\left(p^{2}+r^{2}\right)^{3}}+Q \frac{\left(3 p^{2}+r^{2}\right) r^{4} \mu_{\mathcal{X}} \beta_{X}+\left(p^{2}+3 r^{2}\right) p^{4} \mu_{\mathcal{Y}} \beta_{Y}}{p^{4} r^{4}\left(p^{2}+r^{2}\right)^{2}} \\
\epsilon_{2} & =-3 Q^{2}\left(\mu_{\mathcal{X}}-\mu_{\mathcal{Y}}\right)^{2} \frac{p^{2}-r^{2}}{\left(p^{2}+r^{2}\right)^{4}}+3 Q \frac{p^{4} \mu_{\mathcal{Y}} \beta_{Y}-r^{4} \mu_{\mathcal{X}} \beta_{X}}{p^{4} r^{4}\left(p^{2}+r^{2}\right)}
\end{aligned}
$$

The terms proportional to $Q^{2}\left(\mu_{\mathcal{X}}-\mu_{\mathcal{Y}}\right)^{2}$ are characteristic of this extended Ansatz. So, we recognize at least two interesting cases:

\footnotetext{
${ }^{8}$ Even if we work with Maxwell-Chern-Simons equations, the Maxwellian form of $T_{\nu}^{\mu}$ remains valid. This is because the Chern-Simons term in the action, $F \wedge F \wedge A$, does not contain the metric; thus it does not contribute to $T_{\nu}^{\mu}$.

${ }^{9}$ Equation (59) also accepts the solution $f=-\beta p^{2}+\gamma, g=\beta r^{2}+\delta$; however this solution is already present in $X_{v a c}, Y_{v a c}$ through the integration constants $\alpha, m, n$.
} 
i) If $\beta_{X}=0=\beta_{Y}$, then the eigenvalues of $G^{\mu}{ }_{\nu}+\Lambda \delta^{\mu}{ }_{\nu}$ coincide with those of $8 \pi G T^{\mu}{ }_{\nu}$ (i.e., Einstein equations are verified) provided that

$$
\left(\mu_{\mathcal{X}}-\mu_{\mathcal{Y}}\right)^{2}=\frac{4 G}{3}
$$

Thus the rotating "pointlike" charged solution to Einstein-Maxwell-Chern-Simons equations is obtained only for a specific value of the Chern-Simons coupling constant. The CCLP solution (48) is a particular choice of $\mu_{\mathcal{X}}, \mu_{\mathcal{Y}}$ satisfying the condition (64). However, each choice of $\mu_{\mathcal{X}}, \mu_{\mathcal{Y}}$ fulfilling Eq. (64) still could imply a different geometry. In fact the Kretschmann invariant depends not only on $\mu_{\mathcal{X}}-\mu_{\mathcal{Y}}$ but on $\mu_{\mathcal{X}}, \mu_{\mathcal{Y}}$ in a separate way. For instance, in the simplest case $\lambda=m=n=0$ it results

$$
\begin{aligned}
R_{\lambda \rho}^{\mu \nu} R_{\mu \nu}^{\lambda \rho}= & \frac{4 Q^{2}\left(\mu_{\mathcal{X}}-\mu_{\mathcal{Y}}\right)^{2}}{\left(p^{2}+r^{2}\right)^{8}}\left[\frac{48\left(a^{2}+b^{2}\right)\left(p^{4}-r^{4}\right)^{2}}{p^{2}-r^{2}}\right. \\
& \left.+192\left(a b\left(p^{2}+r^{2}\right)+Q\left(p^{2} \mu_{\mathcal{X}}+r^{2} \mu_{\mathcal{Y}}\right)\right)^{2}+Q^{2}\left(\mu_{\mathcal{X}}-\mu_{\mathcal{Y}}\right)^{2}\left(28 p^{2} r^{2}-65\left(p^{4}+r^{4}\right)\right)\right] .
\end{aligned}
$$

So different values of $\mu_{\mathcal{X}}, \mu_{\mathcal{Y}}$, subjected to the condition (64), lead to different values of the Kretschmann invariant. This is a good indication to think that we have found a one-parametric family of geometries. However, the certainty must come from a proper global analysis of the involved solutions.

ii) If $\beta_{X}=\beta_{Y}$ and $\mu_{\mathcal{X}}=\mu_{\mathcal{Y}}$, then the eigenvalues become

$$
\begin{aligned}
-\epsilon_{0} & =-\epsilon_{3}=\epsilon_{1}=\epsilon_{4}=Q \quad \mu_{\mathcal{X}} \beta_{X} \frac{p^{2}+r^{2}}{p^{4} r^{4}}, \\
\epsilon_{2} & =3 Q \mu_{\mathcal{X}} \beta_{X} \frac{p^{2}-r^{2}}{p^{4} r^{4}} .
\end{aligned}
$$

It would be interesting to look for sources matching this eigenvalue structure.

\section{FURTHER EXTENSIONS OF PLEBAŃSKI-DEMIAŃSKI ANSATZ}

Once we have understood the mechanism to make the CCLP-like solutions work, we can try extending this mechanism to search for more solutions. For instance, we could further extent the Ansatz (42) by considering that $\Omega^{2}$ could have also components $d r$ and $d p$ :

$$
\boldsymbol{\Omega}^{2}=\omega^{2}-\frac{p^{2} r^{2}}{a b\left(p^{2}+r^{2}\right)}\left(\mathcal{Y}(r, p) \omega^{0}+\mathcal{X}(r, p) \omega^{1}\right)-\frac{p^{2} r^{2}}{a b}(\mathcal{Z}(r, p) d r+\mathcal{W}(r, p) d p),
$$

which not only forces the replacements (49) and (50) in the inverse metric, but the replacement of vectors $\partial / \partial r$ and $\partial / \partial p$ with

$$
\begin{aligned}
& \mathbf{V}_{3}=\frac{\partial}{\partial r}+\mathcal{Z}(r, p) \mathbf{v}_{2} \\
& \mathbf{V}_{4}=\frac{\partial}{\partial p}+\mathcal{W}(r, p) \mathbf{v}_{2}
\end{aligned}
$$

Thus the inverse metric becomes

$$
\mathbf{g}^{-1}=-\frac{\mathbf{V}_{0} \otimes \mathbf{V}_{0}}{\left(p^{2}+r^{2}\right) Y(r)}+\frac{\mathbf{V}_{1} \otimes \mathbf{V}_{1}}{\left(p^{2}+r^{2}\right) X(p)}+\frac{\mathbf{v}_{2} \otimes \mathbf{v}_{2}}{p^{2} r^{2}}+\frac{Y(r)}{p^{2}+r^{2}} \mathbf{V}_{3} \otimes \mathbf{V}_{3}+\frac{X(p)}{p^{2}+r^{2}} \mathbf{V}_{4} \otimes \mathbf{V}_{4} .
$$

The determinant of the metric remains independent of $X, Y, \mathcal{X}, \mathcal{Y}, \mathcal{W}, \mathcal{Z}$. The potential (36) still satisfies MaxwellChern-Simons equations (56) for the choices (55), and its energy-momentum tensor is

$$
8 \pi G T_{\nu}^{\mu} \frac{\partial}{\partial x^{\mu}} \otimes d x^{\nu}=-\frac{4 G Q^{2}}{\left(p^{2}+r^{2}\right)^{3}}\left[\mathbf{V}_{\widehat{0}} \otimes \omega^{\widehat{0}}+\mathbf{V}_{3} \otimes d r-\mathbf{V}_{\widehat{1}} \otimes \omega^{\widehat{1}}-\mathbf{V}_{4} \otimes d p+\frac{p^{2}-r^{2}}{p^{2}+r^{2}} \mathbf{V}_{\widehat{2}} \otimes \mathbf{\Omega}^{\widehat{2}}\right]
$$


The separability of $\left(p^{2}+r^{2}\right) \mathbf{g}^{-1}$ is guaranteed by choosing

$$
\mathcal{W}(r, p)=w(p), \quad \mathcal{Z}(r, p)=z(r)
$$

Although the metric is no longer diagonal by blocks in the chart $(t, \phi, \psi, r, p), R_{r}^{p}$ is still zero. However the Ricci tensor $R_{\nu}^{\mu}$ becomes non-linear in $X, Y$; its form is complicated enough to suggest that this strategy will not be successful in getting solutions to Einstein-Maxwell-Chern-Simons equations for other values of the Chern-Simons coupling constant (however, see Ref. 21] for the slowly rotating case).

As a different try, we will invert the procedure of the previous section. Instead of changing $\omega^{2}$ in the metric (10), we will change $\mathbf{v}_{2}$ in the inverse metric (3). This is a rather obvious strategy, once one realizes that $\mathbf{g}$ and $\mathbf{g}^{-1}$ are on an equal footing in the requirements to keep $R_{r p}=0$. If $\mathbf{v}_{2}$ is substituted with

$$
\mathbf{V}_{2}=\mathbf{v}_{2}-\frac{p^{2} r^{2}}{a b\left(p^{2}+r^{2}\right)}\left(\mathcal{N}(r) \mathbf{v}_{0}+\mathcal{M}(p) \mathbf{v}_{1}\right)
$$

then the determinant of $\mathbf{g}^{-1}$ will be preserved; however the separability of $\left(p^{2}+r^{2}\right) \mathbf{g}^{-1}$ will be lost. The corresponding metric $\mathbf{g}$ can be reached by replacing in (11):

$$
\begin{aligned}
& \Omega^{0}=\omega^{0}+\mathcal{N}(r) \omega^{2}, \\
& \boldsymbol{\Omega}^{1}=\omega^{1}+\mathcal{M}(p) \omega^{2},
\end{aligned}
$$

By keeping the "undeformed" value (45) we are led to

$$
\mathcal{N}(r)=\frac{1}{1+\mu r^{2}}, \quad \mathcal{M}(p)=0
$$

or

$$
\mathcal{N}(r)=0, \quad \mathcal{M}(p)=\frac{1}{1+\mu p^{2}}
$$

Nevertheless, the potential (36) is not a solution to Maxwell-Chern-Simons equations in this geometry.

\section{PURE-RADIATION SOLUTION TO $5 D$ EINSTEIN-MAXWELL EQUATIONS}

We will leave the Plebański-Demiański family of metrics, to show a different type of non-static solutions to $5 D$ Einstein-Maxwell equations in the framework of the Kerr-Schild Ansatz (30). We will still use the Plebański-Demiański

form to introduce a suitable seed metric $\stackrel{o}{g}_{\mu \nu}$. In fact, we will start from the metric (1), as written for the vacuum solutions (9) and (10) with the following choice of constants:

$$
b=0, \quad \alpha=1-a^{2} \lambda, \quad m=\frac{a^{2}}{2}=-n
$$

Therefore the functions $X_{v a c}, Y_{v a c}$ become

$$
X_{v a c}(p)=-\lambda p^{4}, \quad Y_{v a c}(r)=-\lambda r^{4} .
$$

Since both $X_{v a c}, Y_{v a c}$ must be positive in order to have a Lorentzian metric, then one gets that $\lambda$ has to be negative; besides it is $m=-n$, what means that the seed metric is the anti-de Sitter (AdS) geometry in a peculiar chart: $\stackrel{o}{g}_{\mu \nu}=\mathbf{g}_{A d S}$. In such chart, the interval associated with the seed metric is

$$
d s_{A d S}^{2}=\left(p^{2}-r^{2}\right) d u^{2}+p^{2} r^{2}\left(2 d u d \sigma+d w^{2}\right)-\lambda^{-1}\left(p^{2}+r^{2}\right)\left(\frac{d r^{2}}{r^{4}}+\frac{d p^{2}}{p^{4}}\right)
$$

where

$$
u=\frac{\sqrt{-\lambda}(t-a \phi)}{1-a^{2} \lambda}, \quad \sigma=a^{-2}(u-\sqrt{-\lambda} t), \quad w=\frac{\psi}{a} .
$$


As can be seen, the parameter $a$ has been absorbed into the new coordinates; no trace of $a$ remains in the interval (81). With the help of the complex coordinate

$$
\zeta=\frac{1}{2 \sqrt{-\lambda}}\left(\frac{1}{r}+\frac{i}{p}\right)^{2}
$$

the interval reads

$$
d s_{A d S}^{2}=4 \lambda^{-1}(\zeta-\bar{\zeta})^{-2}\left[d u(\sqrt{-\lambda}(\zeta+\bar{\zeta}) d u+2 d \sigma)+d w^{2}+d \zeta d \bar{\zeta}\right]
$$

or using the real and imaginary parts of $\zeta=\chi+i y$,

$$
d s_{A d S}^{2}=-\lambda^{-1} y^{-2}\left[d u(2 \sqrt{-\lambda} \chi d u+2 d \sigma)+d w^{2}+d \chi^{2}+d y^{2}\right]
$$

The metric inside the square brackets is flat. In fact, in the chart $(\tau, w, x, y, z)$ such that

$$
u=\tau+z, \quad \sigma=\frac{\lambda(\tau+z)^{3}}{3}-\frac{(\tau-z)}{2}-\sqrt{-\lambda}(\tau+z) x, \quad \chi=x+\frac{\sqrt{-\lambda}(\tau+z)^{2}}{2},
$$

the interval reads

$$
d s_{A d S}^{2}=-\lambda^{-1} y^{-2}\left[-d \tau^{2}+d w^{2}+d x^{2}+d y^{2}+d z^{2}\right]
$$

which is one of known forms the $A d S$ metric can adopt (see Sec. 5.3 in Ref. [22]).

Coming back to the chart $(u, \sigma, w, r, p)$, let us introduce the electromagnetic potential

$$
\mathbf{A}=\frac{A(u)}{p^{2} r^{2}} \mathbf{n}
$$

where $\mathbf{n} \equiv d u$ is the null 1 -form of components

$$
n_{\mu}=\{1,0,0,0,0\} .
$$

The electromagnetic potential (88) fulfills the Maxwell equations not only in the geometry (81) but in any metric having the Kerr-Schild form

$$
\mathbf{g}=\mathbf{g}_{A d S}+f(u, r, p) \mathbf{n} \otimes \mathbf{n} .
$$

This solution is a pure-radiation field, since $T_{\nu}^{\mu}$ has the form 10

$$
T_{\nu}^{\mu}=\frac{-\lambda A(u)^{2}}{\pi p^{4} r^{4}} n^{\mu} n_{\nu}
$$

On the other hand, the Einstein tensor for the metric (90) is

$$
G_{\nu}^{\mu}+\Lambda \delta_{\nu}^{\mu}=-2 \lambda\left(f-\frac{1}{4\left(p^{2}+r^{2}\right)}\left[p^{3} \frac{\partial}{\partial p}\left(p \frac{\partial f}{\partial p}\right)+r^{3} \frac{\partial}{\partial r}\left(r \frac{\partial f}{\partial r}\right)\right]\right) n^{\mu} n_{\nu} .
$$

The fact that both the energy-momentum tensor (91) and the Einstein tensor (92) have the same structure implies that the Kerr-Schild Ansatz is successful in this case, because there is a sole equation to be satisfied by the unknown function $f(u, r, p)$. In fact, Einstein-Maxwell equations are satisfied by choosing 11

$$
f(u, r, p)=-\frac{2}{7} G A(u)^{2} \frac{\left(p^{2}+r^{2}\right)^{2}}{p^{6} r^{6}} .
$$

10 The general solution for Maxwell equations in the considered metric (81), (90) is $\mathbf{A}_{\kappa}=p^{-1} r^{-1}\left(G_{\kappa}(u) J_{1}\left(\kappa p^{-1}\right)+H_{\kappa}(u) Y_{1}\left(\kappa p^{-1}\right)\right)$ $\left(P_{\kappa}(u) I_{1}\left(\kappa r^{-1}\right)+Q_{\kappa}(u) K_{1}\left(\kappa r^{-1}\right)\right) \mathbf{n}$, but it does not guarantee the pure-radiation form of the energy-momentum tensor.

11 Actually Einstein-Maxwell-Chern-Simons equations are fulfilled too. On the one hand, the Chern-Simons term $\epsilon^{\nu \lambda \rho \alpha \beta} F_{\lambda \rho} F_{\alpha \beta}$ vanishes for potentials like [88), since the only non-null independent components of the field tensor are $F_{u r}$ and $F_{u p}$. On the other hand, the Chern-Simons coupling does not contribute to the energy-momentum tensor, as mentioned in Footnote 8 
Besides, the function $f$ can be added with a homogeneous solution of Einstein equations, like

$$
f_{v a c}=\frac{\beta(u)}{p^{2} r^{2}}+\frac{\gamma(u) p^{8} r^{8}}{\left(p^{2}+r^{2}\right)^{5}}+\delta(u) p^{2} r^{2}+\kappa(u) \frac{\left(p^{2}+r^{2}\right)^{3}}{p^{4} r^{4}}+\frac{\varepsilon(u)}{p^{4} r^{4}}\left(\frac{3\left(p^{2}+r^{2}\right)^{2}}{14 p^{2} r^{2}}-1\right)
$$

which generates vacuum solutions representing exact gravitational waves associated with the null 1-form $n_{\mu} 12$ Actually the (linear) homogeneous equation for $f_{\text {vac }}$ can be solved by separating variables; the general solution is obtained by linearly combining the solutions

$$
f_{\text {vac } \nu}=\left(C_{\nu}(u) J_{2}\left(\nu p^{-1}\right)+D_{\nu}(u) Y_{2}\left(\nu p^{-1}\right)\right)\left(E_{\nu}(u) I_{2}\left(\nu r^{-1}\right)+F_{\nu}(u) K_{2}\left(\nu r^{-1}\right)\right)
$$

where $C_{\nu}, D_{\nu}, E_{\nu}, F_{\nu}$ are arbitrary functions of $u$, and $\nu$ is a separation constant.

In sum, we have obtained an electrovacuum solution whose metric is conformal to a $p p$ wave in $5 D$. In fact, according to Eqs. (85) and (90) the interval is

$$
d s^{2}=-\lambda^{-1} y^{-2}\left[d u\left(\left(2 \sqrt{-\lambda} \chi-\lambda y^{2} f\right) d u+2 d \sigma\right)+d w^{2}+d \chi^{2}+d y^{2}\right],
$$

which belongs to the class of metrics studied by Kundt [23], and has the form of Siklos metric [24] (see also Refs. [25], [26]). However the functions (93 [95) are characteristic of five dimensions. Concerning the ppwave metric inside the bracket of Eq. (96), the 1-form (89) has zero covariant derivative whatever the function $f$ is, and is a null vector of the respective Weyl tensor.

\section{CONCLUSIONS}

Plebański-Demiański-like Ansatz (1) does not contain the solution to Einstein-Maxwell equations for a rotating "pointlike" charge in five dimensions. The reason can be traced to the lack of agreement between the eigenvalues of the energy-momentum tensor and those belonging to the Einstein tensor (37) in such an Ansatz, even though the eigenvector structures do coincide. However, the Plebański-Demiański Ansatz can be properly extended to obtain a wider framework where the eigenvector-eigenvalue structure of the energy-momentum and Einstein tensors can be matched, as shown in Section IV] In this extended Ansatz the CCLP solution is found. Remarkably, the CCLP geometry is just one in a set of solutions satisfying Einstein-Maxwell-Chern-Simons equations for the electromagnetic potential (36) and the coupling constant $\mu_{\mathcal{X}}-\mu_{\mathcal{Y}}=2 \sqrt{G / 3}$ in Eq. (56). In fact, this constraint on $\mu_{\mathcal{X}}, \mu_{\mathcal{Y}}$ still leaves alive many different solutions fulfilling the equations, as evidenced by the fact that the Kretschmann invariant in Eq. (65) depends independently on $\mu_{\mathcal{X}}$ and $\mu_{\mathcal{Y}}$. Whether they are different geometries or not should be elucidated through the analysis of the global properties of these solutions.

Although we tried other extensions of Plebański-Demiański Ansatz, we did not succeed in getting solutions sourced by a rotating pointlike charge for other values of the Chern-Simons coupling constant. Instead we have obtained a family of gravitational waves depending on three coordinates, with and without a pure-radiation electromagnetic field. These geometries are described by the interval (96), where $f$ can be substituted with a combination of the functions (93), (94), and (95).

\section{ACKNOWLEDGMENTS}

The author is indebted to Nathalie Deruelle for proposing and encouraging this research, and the Université Paris Diderot for its hospitality and the financial support granted by the Chaire Alicia Moreau. The author also thanks A. Anabalón, M. Cárdenas, O. Fuentealba, G. Giribet, and F. Julié for helpful comments.

\footnotetext{
12 The term $\delta(u) p^{2} r^{2}$ should be absorbable by changing coordinates, since it does not take part in the Riemann tensor.
} 
[1] R.P. Kerr, Phys. Rev. Lett. 11 (1963), 237-238.

[2] R.P. Kerr and A. Schild, A new class of vacuum solutions of the Einstein field equations, in Atti del Convegno sulla Relativita Generale: Problemi dell'Energia e Onde Gravitazionali (Fourth Centenary of Galileo's Birth), ed. by G. Barbèra (Firenze, 1965); republished in Gen. Relativ. Gravit. 41 (2009), 2485-2499.

[3] G.C. Debney, R.P. Kerr, and A. Schild, J. Math. Phys. 10 (1969), 1842-1854.

[4] J.F. Plebanski and M. Demianski, Ann. Phys. (N.Y.) 98 (1976), 98-127.

[5] J. Podolský, O. Hruška, and J.B. Griffiths, Class. Quantum Grav. 35 (2018), 165011. arXiv:1804.01519 [gr-qc]

[6] R.C. Myers and M.J. Perry, Ann. Phys. (N.Y.) 172 (1986), 304-347.

[7] G.W. Gibbons, H. Lü, D.N. Page, and C.N. Pope, J. Geom. Phys. 53 (2005), 49-73. hep-th/0404008

[8] Z.-W. Chong, G.W. Gibbons, H. Lü, and C.N. Pope, Phys. Lett. B 609 (2005), 124-132. hep-th/0405061

[9] Z.-W. Chong, M. Cvetič, H. Lü, and C.N. Pope, Phys. Rev. Lett. 95 (2005), 161301. hep-th/0506029

[10] J. Kunz and F. Navarro-Lérida, Phys. Rev. Lett. 96 (2006), 081101. hep-th/0510250

[11] J.L. Blázquez-Salcedo, J. Kunz, F. Navarro-Lérida, and E. Radu, Phys. Rev. D 95 (2017), 064018. arXiv:1610.05282 [gr-qc]

[12] M. Mir and R.B. Mann, Phys. Rev. D 95 (2017), 024005. arXiv:1610.05281 [gr-qc]

[13] S.S. Yazadjiev, Phys. Rev. D 73 (2006), 104007. hep-th/0602116

[14] W. Chen, H. Lü, and C.N. Pope, Class. Quantum Grav. 23 (2006), 5323-5340. hep-th/0604125

[15] P. Davis, H.K. Kunduri, and J. Lucietti, Phys. Lett. B 628 (2005), 275-280. hep-th/0508169

[16] T. Dereli and M. Gürses, Phys. Lett. B 171 (1986), 209-211.

[17] M. Gürses and F. Gürsey, J. Math. Phys. 16 (1975), 2385-2390.

[18] H. Lü, Jianwei Mei, and C.N. Pope, Class. Quantum Grav. 27 (2010) 075013. arXiv:0806.2204 [hep-th]

[19] H. Lü, Jianwei Mei, and C.N. Pope, Nuclear Physics B 806 (2009), 436-455. arXiv:0804.1152 [hep-th]

[20] L.D. Landau and E.M. Lifshitz, The Classical Theory of Fields, Course of Theoretical Physics Vol. 2, Pergamon Press (Oxford, 1994).

[21] A.N. Aliev and D.K. Çiftçi, Phys. Rev. D 79 (2009), 044004. arXiv:0811.3948 [hep-th]

[22] J.B. Griffiths and J. Podolský, Exact Space-Times in Einstein's General Relativity, CUP (Cambridge, 2012).

[23] W. Kundt, Proc. Roy. Soc. A 270 (1962), 328-334.

[24] S.T.C. Siklos, in Galaxies, axisymmetric systems and relativity, essays presented to W. B. Bonnor on his 65 th birthday, CUP (Cambridge, 1985).

[25] M. Wyman and R. Trollope, J. Math. Phys. 6(1965), 1995-2007.

[26] R.G. McLenaghan, N. Tariq, and B.O.J. Tupper, J. Math. Phys. 16 (1975), 829-831. 